\title{
Assessment of various parameters to improve MALDI-TOF MS reference spectra libraries constructed for the routine identification of filamentous fungi
}

Anne-Cécile Normand ${ }^{1 *}$, Carole Cassagne ${ }^{1,2}$, Stéphane Ranque ${ }^{1,2}$, Coralie L'Ollivier ${ }^{1}$, Patrick Fourquet ${ }^{3}$, Sam Roesems ${ }^{4}$, Marijke Hendrickx ${ }^{4}$ and Renaud Piarroux ${ }^{1,2}$

\begin{abstract}
Background: The poor reproducibility of matrix-assisted desorption/ionization time-of-flight (MALDI-TOF) spectra limits the effectiveness of the MALDI-TOF MS-based identification of filamentous fungi with highly heterogeneous phenotypes in routine clinical laboratories. This study aimed to enhance the MALDI-TOF MS-based identification of filamentous fungi by assessing several architectures of reference spectrum libraries.

Results: We established reference spectrum libraries that included 30 filamentous fungus species with various architectures characterized by distinct combinations of the following: i) technical replicates, i.e., the number of analyzed deposits for each culture used to build a reference meta-spectrum (RMS); ii) biological replicates, i.e., the number of RMS derived from the distinct subculture of each strain; and iii) the number of distinct strains of a given species. We then compared the effectiveness of each library in the identification of 200 prospectively collected clinical isolates, including 38 species in 28 genera.

Identification effectiveness was improved by increasing the number of both RMS per strain $\left(p<10^{-4}\right)$ and strains for a given species $\left(p<10^{-4}\right)$ in a multivariate analysis.

Conclusion: Addressing the heterogeneity of MALDI-TOF spectra derived from filamentous fungi by increasing the number of RMS obtained from distinct subcultures of strains included in the reference spectra library markedly improved the effectiveness of the MALDI-TOF MS-based identification of clinical filamentous fungi.
\end{abstract}

Keywords: MALDI-TOF MS, Filamentous fungi, Molds, Reference spectra library

\section{Background}

The identification of mold in the clinical laboratory is classically based on macroscopic and microscopic examination of the colonies grown on mycological culture media. It is a slow and complex process requiring highly skilled mycologists, and misidentifications may occur, even in experienced reference laboratories [1]. Additionally, some distinct species, which are identified via DNA sequence analysis, are morphologically indistinguishable [2-4]. Therefore, multilocus DNA sequence analysis represents the recommended approach to accurately

\footnotetext{
* Correspondence: anne-cecile.normand@ap-hm.fr

'AP-HM, Parasitologie-Mycologie, CHU Timone, 13005, Marseille, France Full list of author information is available at the end of the article
}

identify these microorganisms. Nevertheless, the DNA sequence-based identification of filamentous fungi is primarily limited by the following: i) low DNA extraction yields because mold cells are difficult to lyse, ii) the presence of PCR inhibitors, iii) the presence of misidentified sequences in non-curated public DNA sequence databases, and iv) the cost and time required for sequencing. Currently, only some clinical laboratories routinely use a molecular approach for microorganism identification, which is primarily due to the cost and application constraints $[5,6]$.

Recently, matrix-assisted desorption/ionization timeof-flight (MALDI-TOF) mass spectrometry (MS) has been applied to rapidly identify bacteria and yeasts in

\section{Biomed Central}


the clinical microbiology laboratory setting [7]. This technique is used to analyze microorganism content (primarily ribosomal proteins), thereby generating a spectrum that is considered the fingerprint of the microorganism [8]. Using this technique, the identification of an unknown organism is performed by comparing the corresponding spectrum to a reference library of spectra. When establishing a reference library for microbial identification purposes, many authors have used reference mass spectra, sometimes referred to as "metaspectra" or "superspectra", which are generated by combining the results of a various number of individual spectra corresponding to technical replicates of a given sample. Previous studies have indicated that MS could be used to identify various filamentous fungi taxa of clinical interest, including Fusarium spp [9-11], dermatophytes [12,13], Aspergillus spp [14,15], and Pseudallescheria/Scedosporium spp [16]; those of industrial interest, including Penicillium spp $[17,18]$, Verticillium spp [19], and Trichoderma spp [20]; and various filamentous fungal contaminants frequently isolated in the clinical laboratory [21,22].

The heterogeneous morphological phenotypes of filamentous fungi affect the identification process. As shown in Figure 1, the same heterogeneity exists for MALDITOF mass spectra, between different strains of the same species as well as between subcultures of the same strain, which negatively impacts the reproducibility of the spectra. To troubleshoot this issue, we accounted for this heterogeneity during the establishment of the RMS library (MSL). We hypothesized that MS identification effectiveness could be enhanced by increasing both the number of reference meta spectra (RMS) of a given strain included in the reference library and the number of deposits used to generate each RMS. The primary objective of this study was to test the effectiveness of distinct reference spectra library architectures for the MALDI-TOF MS-based identification of filamentous fungi. More precisely, we assessed the influence on identification effectiveness of the following: i) the number of technical replicates, i.e., the number of analyzed deposits (spots) from one culture used to generate an RMS; ii) the number of biological replicates, i.e., the number of RMS derived from distinct subcultures for each strain; and iii) the number of distinct strains of one species used to construct the library.

\section{Results}

\section{Phenotypic and genotypic identification of clinical} isolates

The results of the classical and DNA sequence-based identification of 200 clinical isolates (Table 1) were applied

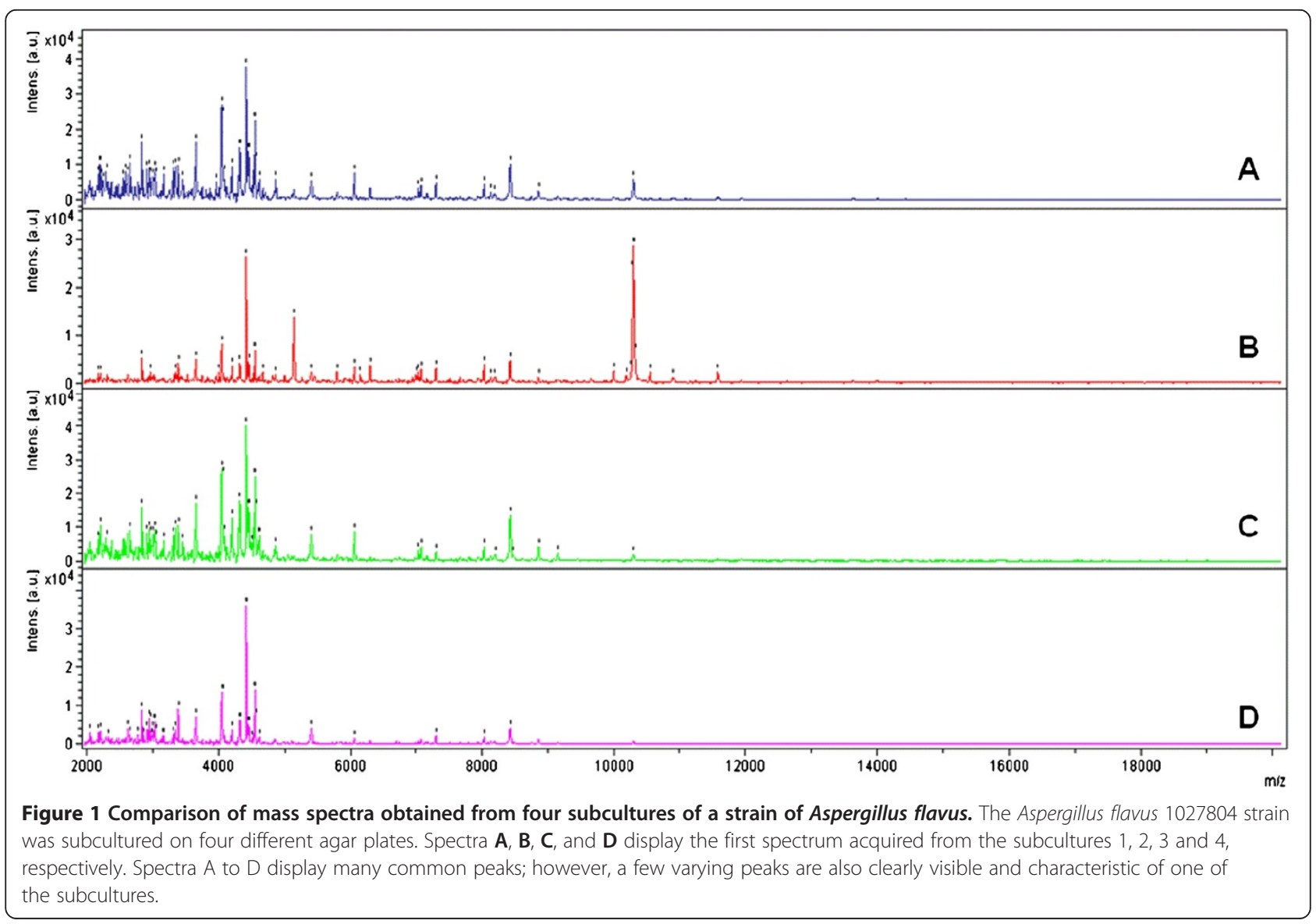


Table 1 Identification of the $\mathbf{2 0 0}$ clinical isolates included in the study

\begin{tabular}{|c|c|c|}
\hline Species & $\begin{array}{l}\text { Number of } \\
\text { Isolates }\end{array}$ & $\begin{array}{l}\text { Corresponding RMS } \\
\text { in the MSLs }\end{array}$ \\
\hline Acremonium sp. & 1 & no \\
\hline Alternaria alternata & 10 & yes \\
\hline Aspergillus alliaceus & 2 & no \\
\hline Aspergillus clavatus & 1 & no \\
\hline Aspergillus flavus & 8 & yes \\
\hline Aspergillus fumigatus & 82 & yes \\
\hline Aspergillus melleus & 1 & no \\
\hline Aspergillus nidulans & 1 & yes \\
\hline Aspergillus niger & 12 & yes \\
\hline Aspergillus oryzae & 1 & no \\
\hline Aspergillus sydowii & 1 & no \\
\hline Aspergillus terreus & 11 & yes \\
\hline Beauveria bassiana & 1 & yes \\
\hline Chaetomium globosum & 1 & no \\
\hline Fomitopsis ostreiformis & 1 & no \\
\hline Fusarium oxysporum & 2 & yes \\
\hline Geotrichum candidum & 2 & no \\
\hline Hamigera avellanea & 1 & no \\
\hline Hypocrea jecorina & 1 & no \\
\hline Microsporum audouinii & 10 & yes \\
\hline Microsporum canis & 1 & yes \\
\hline Onychocola canadensis & 1 & no \\
\hline Penicillium aurantiogriseum & 1 & yes \\
\hline Penicillium chrysogenum & 7 & yes \\
\hline Penicillium citrinum & 1 & no \\
\hline Penicillium funiculosum & 1 & no \\
\hline Penicillium glabrum & 4 & no \\
\hline Penicillium oxalicum & 1 & no \\
\hline Penicillium pinophilum & 1 & no \\
\hline Penicillium variotii & 1 & yes \\
\hline Penicillum spinulosum & 2 & no \\
\hline Rhizopus oryzae & 3 & yes \\
\hline $\begin{array}{l}\text { Scedosporium } \\
\text { apiospermium }\end{array}$ & 8 & yes \\
\hline Trichoderma atroviridae & 1 & no \\
\hline Trichoderma viridae & 1 & no \\
\hline $\begin{array}{l}\text { Trichophyton } \\
\text { mentagrophytes }\end{array}$ & 2 & yes \\
\hline Trichophyton rubrum & 8 & yes \\
\hline Trichophyton soudanense & 6 & yes \\
\hline
\end{tabular}

RMS: reference mass spectrum in the mass spectra library (MSL) constructed from several raw spectra. to classify the isolates into two groups: isolates included and isolates excluded from the MSL. The MS results of both groups are summarized in Table 2. The isolates belonged to 28 different genera and 38 different species. Moreover, 174 isolates corresponded to 18 species, which were represented among those used to construct the eight libraries, whereas the 26 remaining isolates belonged to 20 species that were not represented in the libraries.

\section{Reference MS library validation}

All 104 spectra derived from the 26 clinical isolates for which the species was not included in the seven MS libraries (4 raw spectra per clinical isolate) yielded low Log Scores (LS) ranging from 0.45 to 1.79 (only 1/104 spectra yielded LS>1.7: Penicillium aurantiogriseum identified instead of Geotrichum candidum) regardless of the library utilized, which is markedly below the manufacturer recommended threshold of 2.00 for a valid identification. The number of correct identifications among the 706 remaining spectra (i.e., corresponding to the species included in the libraries) and the corresponding LS values were statistically different depending on the mass spectra library used for identification (Figures 2 and 3). Notably, the number of identifications concordant with the molecular biology or microscopic identification and LS values significantly increased when the library included an increased number of both RMS per strain and strains per species. In contrast, constructing RMS from 40 raw spectra (B5) instead of 10 raw spectra or reducing the number of raw spectra used to build RMS of the B1 library from 10 to 4 (B0) failed to significantly alter the performance of the identification process (Table 3, Figure 3). Overall, the best results were obtained using library B7, which involved the combination of the highest number of RMS per strain and the highest number of strains per species. Using this library, we obtained 611 (87\%) concordant identifications, with LS values higher than 1.700 in $80.85 \%$ (494/611) of the cases and LS values higher than 2.000 in 50.90\% (311/ 611 ) of the cases. Conversely, all 91 (13\%) nonconcordant identifications exhibited LS values less than 1.700 , a value under which the results of LS identification should not be taken in account. These results were dramatically improved compared with those obtained using library B1, which included only one isolate per species and one subculture per isolate. Indeed, using the B1 library, we only obtained 449 (64\%) concordant identifications, $40.09 \%$ of which displayed LS values higher than $1.7(180 / 449)$ and only 15.59\% were higher than 2.000 (70/449). Modulation of the MSP creation parameters, while considering the B1 library, tended to show that the performance of the database could be improved by an increased peak frequency minimum, regarding the number of concordant identifications and the Log Score 
Table 2 Details of the MS-based identification results of the 200 clinical isolates included in the study

\begin{tabular}{|c|c|c|c|c|c|c|c|c|}
\hline & \multicolumn{8}{|c|}{ Mass spectra libraries } \\
\hline & $\mathrm{BO}$ & B1 & B2 & B3 & B4 & B5 & B6 & B7 \\
\hline \multicolumn{9}{|c|}{ Isolates included in the MSLs $(n=174)$} \\
\hline Nb. of concordant identifications & 481 & 449 & 495 & 521 & 494 & 475 & 586 & 611 \\
\hline Median value of concordant LS1 values & 1.59 & 1.58 & 1.65 & 1.73 & 1.67 & 1.67 & 1.99 & 2.02 \\
\hline $\mathrm{Nb}$. of concordant values with LS1>1.7 & 182 & 180 & 222 & 282 & 225 & 225 & 443 & 494 \\
\hline Percentage of concordant values with $L S 1>1.7$ & 37.8 & 40.1 & 44.8 & 54.1 & 45.5 & 47.4 & 75.6 & 80.9 \\
\hline Range of concordant LS1 values & $\begin{array}{l}0.49- \\
2.39\end{array}$ & $\begin{array}{l}0.29- \\
2.45\end{array}$ & $\begin{array}{l}0.50- \\
2.45\end{array}$ & $\begin{array}{l}0.66- \\
2.57\end{array}$ & $\begin{array}{l}0.18- \\
2.44\end{array}$ & $\begin{array}{l}0.70- \\
2.44\end{array}$ & $\begin{array}{l}0.60- \\
2.57\end{array}$ & $\begin{array}{l}0.77- \\
2.57\end{array}$ \\
\hline $\mathrm{Nb}$. of non-concordant identifications & 225 & 257 & 211 & 184 & 212 & 231 & 119 & 95 \\
\hline Median value of non-concordant LS1 values & 0.99 & 1.07 & 1.1 & 1.23 & 1.15 & 1.07 & 1.26 & 1.28 \\
\hline Range of non-concordant LS1 values & $\begin{array}{l}0.29- \\
1.44\end{array}$ & $\begin{array}{l}0.14- \\
1.55\end{array}$ & $\begin{array}{l}0.27- \\
1.58\end{array}$ & $\begin{array}{l}0.43- \\
1.58\end{array}$ & $\begin{array}{l}0.25- \\
1.85\end{array}$ & $\begin{array}{l}0.14- \\
1.52\end{array}$ & $\begin{array}{l}0.65- \\
1.69\end{array}$ & $0.69-$ \\
\hline \multicolumn{9}{|c|}{ Isolates not included in the MSLs $(n=26)$} \\
\hline $\mathrm{Nb}$. of concordant identifications & 0 & 0 & 0 & 0 & 0 & 0 & 0 & 0 \\
\hline Median values of concordant LS1 values & - & - & - & - & - & - & - & - \\
\hline $\begin{array}{l}\text { Minimum and maximum values of the concordant } \\
\text { LS1 }\end{array}$ & - & - & - & - & - & - & - & - \\
\hline $\mathrm{Nb}$. of non-concordant identifications & 104 & 104 & 104 & 104 & 104 & 104 & 104 & 104 \\
\hline Median values of non-concordant LS1 values & 1.02 & 1.09 & 1.18 & 1.24 & 1.22 & 1.14 & 1.31 & 1.33 \\
\hline Range of non-concordant LS1 values & $\begin{array}{l}0.50- \\
1.39\end{array}$ & $\begin{array}{l}0.45- \\
1.43\end{array}$ & $\begin{array}{l}0.46- \\
1.44\end{array}$ & $\begin{array}{l}0.56- \\
1.56\end{array}$ & $\begin{array}{l}0.52- \\
1.54\end{array}$ & $\begin{array}{l}0.54- \\
1.49\end{array}$ & $\begin{array}{l}0.76- \\
1.79\end{array}$ & $\begin{array}{l}0.88- \\
1.79\end{array}$ \\
\hline
\end{tabular}

Concordant LS1: LS value for the first concordant identification with the library; non-concordant LS1: LS value for the first non-concordant identification with the library; Nb.: number.

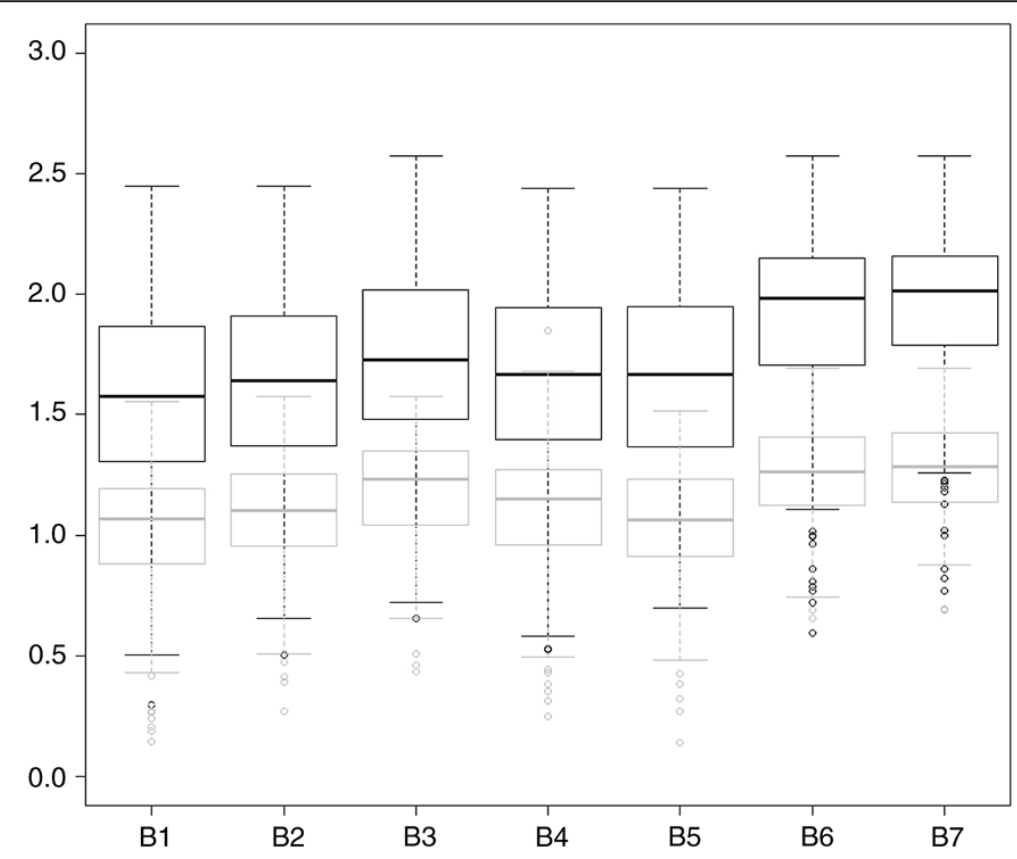

Figure 2 Distribution of the LS1 values. Box-and-whisker diagrams of the LS1 values associated with the concordant mass spectral identifications (black) and the non-concordant identifications (gray) obtained using the seven different mass spectrum libraries tested (B1 to B7). The lower and upper portions of the box represent the lower and upper quartiles, respectively. The dark band represents the median value. The ends of the whiskers represent the lowest datum included in the 1.5 inter-quartile range (IQR) of the lower quartile and the highest datum included in the 1.5 IQR of the upper quartile. Outlier values are represented by a circle; a.u.: arbitrary unit. 


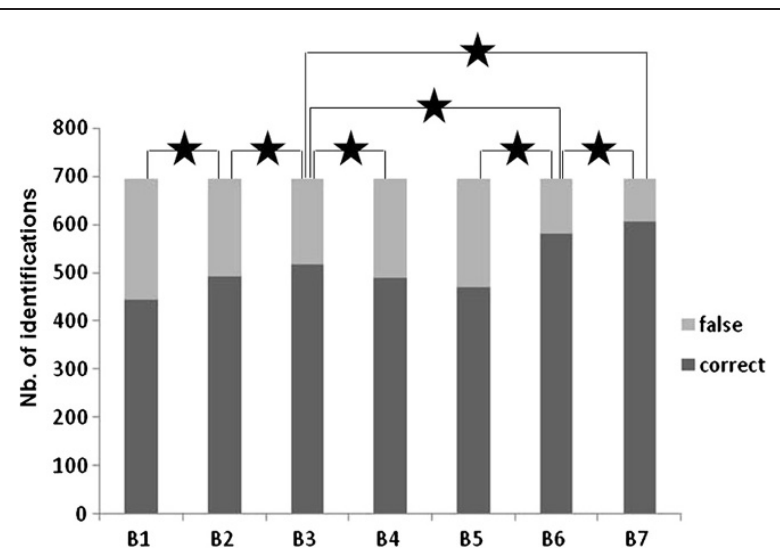

Figure 3 Number of correct and false MALDI-TOF MS-based identifications obtained with the seven mass spectral libraries. A bar graph showing the number of concordant and nonconcordant MALDI-TOF MS-based identifications obtained with each of the seven different mass spectral libraries, B1 to B7, for the 174 isolates. The horizontal bar represents the significance of the McNemar's test between the designated MSLs ( $\star p \leq 0.01 ; \mathrm{Nb}$.: number; MSLs, mass spectral libraries).

of the first identification (LS1) mean value. However, when these parameters were applied to the B7 library, we observed the opposite result (Table 4).

Considering Aspergillus fumigatus isolates separately, the results ranged from $79 \%$ (B0/B1) to $97 \%$ (B7) concordant identifications, whereas for other species, the percentage of concordant identification ranged from $56 \%$ (B0/B1) to $79 \%$ (B7) (Table 3). Finally, the identification of a clinical isolate, regardless of the species, was not improved by creating metaspectra (MSP) of the 4 spectra for the comparison of the various libraries (Table 3).

The multivariate analysis findings (Table 5) indicate that concordant identification rates increased significantly with the number of both RMS per strain and raw spectra per RMS. Similarly, the LS values significantly increased $\left(\mathrm{p}<10^{-4}\right)$ with the independent effect of the numbers of RMS per strain and raw spectra per RMS (data not shown). The independent effect of the number of raw spectra per RMS was weaker than the effect of the number of RMS per strain. The percentage of concordant identifications significantly increased exclusively when the number or raw spectra per RMS exceeded 20 (i.e., 40 raw spectra per RMS).

\section{Discussion}

In contrast with recurrent efforts to improve the reproducibility of the MS-based identification of filamentous fungi by standardizing the pre-treatment procedures, we report the first study aiming to improve identification by comparing the effectiveness of distinct RMS library architectures. However, in a recently published study aiming to identify filamentous fungi using MS, de Carolis et al. [22] have shown that some of the mass spectra data obtained during routine diagnosis matched preferentially with the RMS obtained from either young or mature cultures of the same species. Regarding Scedosporium identification, Coulibaly et al. [16] have shown that both the culture media and the duration of culture had a significant impact on MALDI-TOF assay results. However, the standard recommendation to address problems associated with the heterogeneity of microorganism species is merely to increase the number of strains per species in the library. Our findings confirm this hypothesis; however, it is particularly challenging to increase the number of well-characterized strains included in the RMS library for each fungal species. Numerous species have been described to play a role in human infections and, in many cases, only a single strain or a few strains of the same species are preserved in international collections. In the current study, we demonstrated that increasing the number of mass spectra generated from distinct subcultures of a given strain yields a significant improvement in the process of filamentous fungi identification and can partially offset the relatively low number of specific strains available to construct RMS libraries. Modulating MSP creation parameters yielded discrepant results depending on the database that was taken into account. As the B7 database appears ideal for filamentous fungi identification, Bruker's default parameters for the MSP creation method seem to be more suitable for library construction.

Conversely, the number of spectra derived from a strain $(4,10,20$, or 40$)$ that were used to construct RMS did not result in a marked improvement of the identification performance. This straightforward optimization of RMS library architecture significantly enhanced the identification effectiveness.

In this study, we used quadruplicates of the clinical samples to test the various RMS libraries. By taking only the spectrum with the highest LS value into account, we observed an increased percentage of concordant identifications (e.g., ranging from $87 \%$ to $90 \%$ with library B7). In parallel, using the four clinical replicates to construct an MSP and then compare it to the various libraries did not alter the results but instead tended to complicate the procedure, as this cannot be performed with RTC software during routine analyses.

The use of standardized conditions (incubation time, temperature, and culture medium) [10,15-18] reduces filamentous fungi pleomorphism but does not preclude the heterogeneity of the mass spectra derived from a given isolate. For example, Chen et al. [17] have improved the accuracy of Penicillium identification by assessing the presence or absence of different speciesspecific peaks in the mass spectrum data obtained when analyzing Penicillium spores; however, separating spores from hyphae significantly complicates the pre-processing step. Conversely, some authors have shown that mass spectra heterogeneity is reduced using non-sporulating 
Table 3 The performance of the libraries considering the species and treatment applied to the clinical quadruplicates

\begin{tabular}{|c|c|c|c|c|c|c|c|c|c|c|c|c|c|c|c|c|c|c|c|c|c|c|c|c|c|}
\hline \multirow[b]{2}{*}{ Species } & \multicolumn{9}{|c|}{ Independent spots } & \multicolumn{8}{|c|}{ Best of 4 spots } & \multicolumn{8}{|c|}{ Clinical isolate MSP for 4 spots } \\
\hline & & B0 & B1 & B2 & B3 & B4 & B5 & B6 & B7 & B0 & B1 & B2 & B3 & B4 & B5 & B6 & B7 & BO & B1 & B2 & B3 & B4 & B5 & B6 & B7 \\
\hline \multirow[t]{2}{*}{ Alternaria alternata (10) } & a & 70 & 73 & 80 & 88 & 88 & 83 & 95 & 95 & 60 & 70 & 90 & 90 & 80 & 90 & 100 & 100 & 80 & 80 & 80 & 80 & 80 & 70 & 100 & 100 \\
\hline & $b$ & 1.67 & 1.83 & 1.84 & 1.82 & 1.78 & 1.71 & 1.91 & 1.95 & 1.91 & 1.96 & 1.87 & 1.89 & 1.81 & 1.79 & 1.98 & 2.02 & 1.63 & 1.7 & 1.81 & 1.84 & 1.74 & 1.77 & 1.85 & 1.92 \\
\hline \multirow[t]{2}{*}{ Aspergillus flavus (8) } & $\mathrm{a}$ & 91 & 78 & 81 & 88 & 88 & 94 & 94 & 100 & 88 & 88 & 100 & 100 & 100 & 100 & 100 & 100 & 88 & 75 & 63 & 63 & 75 & 88 & 88 & 100 \\
\hline & $b$ & 1.58 & 1.64 & 1.68 & 1.73 & 1.65 & 1.72 & 1.74 & 2.01 & 1.8 & 1.76 & 1.73 & 1.77 & 1.73 & 1.8 & 1.83 & 2.09 & 1.54 & 1.66 & 1.93 & 1.95 & 1.77 & 1.77 & 2.02 & 2.03 \\
\hline \multirow[t]{2}{*}{ Aspergillus fumigatus (85) } & a & 84 & 79 & 84 & 88 & 86 & 85 & 96 & 97 & 92 & 91 & 91 & 91 & 93 & 89 & 98 & 98 & 88 & 85 & 87 & 87 & 86 & 88 & 99 & 98 \\
\hline & $b$ & 1.58 & 1.59 & 1.67 & 1.77 & 1.7 & 1.71 & 2.03 & 2.04 & 1.69 & 1.69 & 1.77 & 1.87 & 1.78 & 1.82 & 2.13 & 2.14 & 1.58 & 1.6 & 1.67 & 1.76 & 1.69 & 1.64 & 2.05 & 2.08 \\
\hline \multirow[t]{2}{*}{ Aspergillus nidulans (2) } & a & 29 & 14 & 14 & 43 & 57 & 29 & 14 & 43 & 50 & 50 & 50 & 50 & 100 & 50 & 50 & 50 & 50 & 50 & 50 & 50 & 100 & 50 & 50 & 50 \\
\hline & $b$ & 1.37 & 1.89 & 1.89 & 1.56 & 1.53 & 1.39 & 1.89 & 1.82 & 1.58 & 1.89 & 1.89 & 1.89 & 1.52 & 1.49 & 1.89 & 1.89 & 1.64 & 1.62 & 1.62 & 1.63 & 1.41 & 1.14 & 1.63 & 1.83 \\
\hline \multirow[t]{2}{*}{ Aspergillus niger (12) } & $\mathrm{a}$ & 85 & 83 & 81 & 77 & 65 & 63 & 77 & 83 & 92 & 83 & 83 & 83 & 67 & 67 & 83 & 83 & 83 & 83 & 75 & 75 & 75 & 75 & 92 & 83 \\
\hline & $b$ & 1.56 & 1.57 & 1.59 & 1.66 & 1.54 & 1.55 & 1.77 & 1.89 & 1.67 & 1.67 & 1.68 & 1.73 & 1.69 & 1.71 & 1.83 & 1.97 & 1.53 & 1.47 & 1.58 & 1.65 & 1.57 & 1.47 & 1.6 & 1.89 \\
\hline \multirow[t]{2}{*}{ Aspergillus terreus (10) } & a & 28 & 25 & 33 & 35 & 28 & 25 & 55 & 63 & 30 & 30 & 40 & 40 & 40 & 40 & 60 & 70 & 50 & 40 & 50 & 50 & 50 & 40 & 70 & 70 \\
\hline & $b$ & 1.23 & 1.14 & 1.19 & 1.3 & 1.22 & 1.22 & 1.67 & 1.61 & 1.35 & 1.29 & 1.36 & 1.41 & 1.41 & 1.35 & 1.79 & 1.7 & 1.06 & 1.17 & 1.14 & 1.2 & 1.21 & 1.24 & 1.66 & 1.66 \\
\hline \multirow[t]{2}{*}{ Beauveria bassiana (1) } & $\mathrm{a}$ & 0 & 0 & 100 & 100 & 75 & 75 & 75 & 75 & 0 & 0 & 100 & 100 & 100 & 100 & 100 & 100 & 0 & 0 & 0 & 0 & 0 & 0 & 0 & 0 \\
\hline & $b$ & & & 1.2 & 1.2 & 1.05 & 0.93 & 1.24 & 1.26 & & & 1.32 & 1.32 & 1.12 & 1.06 & 1.32 & 1.32 & & & & & & & & \\
\hline \multirow[t]{2}{*}{ Fusarium oxysporum (2) } & a & 100 & 100 & 100 & 100 & 100 & 100 & 100 & 100 & 100 & 100 & 100 & 100 & 100 & 100 & 100 & 100 & 100 & 100 & 100 & 100 & 100 & 100 & 100 & 100 \\
\hline & $b$ & 1.93 & 2.06 & 2.06 & 2.07 & 1.82 & 1.78 & 2.11 & 2.12 & 2 & 2.11 & 2.11 & 2.11 & 1.98 & 2 & 2.16 & 2.17 & 1.97 & 2.06 & 2.06 & 2.06 & 1.79 & 1.9 & 2.06 & 2.06 \\
\hline \multirow[t]{2}{*}{ Microsporum audouinii (10) } & a & 45 & 33 & 30 & 30 & 40 & 33 & 30 & 65 & 60 & 50 & 50 & 50 & 40 & 40 & 50 & 70 & 50 & 50 & 50 & 50 & 30 & 40 & 50 & 80 \\
\hline & $b$ & 1.49 & 1.4 & 1.44 & 1.57 & 1.35 & 1.47 & 1.59 & 1.8 & 1.59 & 1.54 & 1.55 & 1.7 & 1.64 & 1.67 & 1.7 & 1.91 & 1.41 & 1.2 & 1.38 & 1.45 & 1.59 & 1.33 & 1.54 & 1.71 \\
\hline \multirow[t]{2}{*}{ Microsporum canis (1) } & $\mathrm{a}$ & 0 & 0 & 0 & 0 & 0 & 0 & 25 & 50 & 0 & 0 & 0 & 0 & 0 & 0 & 0 & 100 & 0 & 0 & 0 & 0 & 0 & 0 & 0 & 100 \\
\hline & $b$ & & & & & & & 1.17 & 1.51 & & & & & & & & 1.56 & & & & & & & & 1.65 \\
\hline \multirow[t]{2}{*}{ Penicillium aurantiogriseum/chrysogenum (8) } & a & 34 & 34 & 44 & 63 & 41 & 28 & 75 & 75 & 38 & 25 & 50 & 63 & 50 & 38 & 75 & 75 & 50 & 0 & 0 & 0 & 0 & 0 & 38 & 50 \\
\hline & $b$ & 1.7 & 1.59 & 1.58 & 1.88 & 1.64 & 1.88 & 1.98 & 2 & 1.86 & 2.1 & 1.72 & 2.03 & 1.65 & 1.87 & 2.19 & 2.19 & 1.75 & & & & & & 2.07 & 2.11 \\
\hline \multirow[t]{2}{*}{ Paecilomyces variotii (1) } & a & 0 & 0 & 0 & 0 & 0 & 0 & 25 & 25 & 0 & 0 & 0 & 0 & 0 & 0 & 100 & 100 & 0 & 0 & 0 & 0 & 0 & 0 & 0 & 100 \\
\hline & $b$ & & & & & & & 1.2 & 1.28 & & & & & & & 1.2 & 1.28 & & & & & & & & 1.76 \\
\hline \multirow[t]{2}{*}{ Rhizopus oryzae (3) } & $\mathrm{a}$ & 58 & 50 & 58 & 75 & 50 & 58 & 75 & 75 & 67 & 67 & 100 & 100 & 33 & 67 & 100 & 100 & 67 & 67 & 100 & 100 & 67 & 67 & 67 & 67 \\
\hline & $b$ & 1.64 & 2.14 & 2.05 & 2.05 & 1.89 & 1.69 & 2.05 & 2.05 & 2.03 & 2.15 & 1.95 & 2.06 & 2.28 & 1.92 & 2.06 & 2.06 & 1.89 & 2.16 & 1.84 & 1.92 & 2.02 & 1.75 & 2.27 & 2.27 \\
\hline \multirow[t]{2}{*}{ Scedosporium apiospermum (8) } & a & 47 & 44 & 41 & 47 & 44 & 41 & 56 & 66 & 50 & 50 & 50 & 63 & 38 & 38 & 50 & 63 & 50 & 50 & 63 & 63 & 38 & 63 & 63 & 75 \\
\hline & $b$ & 1.53 & 1.33 & 1.45 & 1.56 & 1.59 & 1.52 & 1.62 & 1.67 & 1.69 & 1.63 & 1.65 & 1.71 & 1.96 & 1.81 & 1.84 & 1.9 & 1.97 & 1.83 & 1.88 & 1.9 & 2.26 & 1.81 & 1.96 & 2.02 \\
\hline \multirow[t]{2}{*}{ Trichophyton mentagrophytes (2) } & a & 88 & 63 & 100 & 100 & 88 & 100 & 100 & 100 & 100 & 50 & 100 & 100 & 50 & 100 & 100 & 100 & 100 & 50 & 100 & 100 & 50 & 100 & 100 & 100 \\
\hline & $b$ & 1.91 & 1.93 & 1.9 & 1.93 & 1.8 & 1.67 & 1.94 & 1.94 & 1.92 & 2.37 & 1.97 & 2.01 & 2.44 & 1.74 & 2.01 & 2.01 & 1.84 & 2.35 & 1.96 & 2.01 & 2.47 & 1.69 & 2.01 & 2.01 \\
\hline Trichophyton rubrum (8) & a & 47 & 50 & 53 & 69 & 53 & 53 & 78 & 69 & 50 & 75 & 88 & 75 & 63 & 63 & 88 & 75 & 63 & 50 & 63 & 63 & 63 & 38 & 63 & 50 \\
\hline & $b$ & 1.11 & 1.13 & 1.28 & 1.43 & 1.47 & 1.29 & 1.51 & 1.65 & 1.52 & 1.27 & 1.35 & 1.5 & 1.64 & 1.54 & 1.63 & 1.83 & 1.35 & 1.07 & 1.38 & 1.46 & 1.47 & 1.81 & 1.54 & 1.74 \\
\hline
\end{tabular}


Table 3 The performance of the libraries considering the species and treatment applied to the clinical quadruplicates (Continued)

Trichophyton soudanense (6)

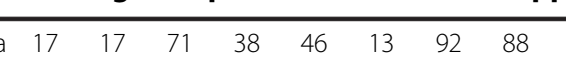

$\begin{array}{lllll}17 & 67 & 50 & 50 & 0\end{array}$

$100 \quad 100$

$\begin{array}{lllllllllllllll}\text { b } & 1.08 & 1.24 & 1.39 & 1.46 & 1.37 & 1.17 & 1.91 & 1.97 & 1.35 & 1.48 & 1.53 & 1.42 & 2 & 2.02\end{array}$

$\begin{array}{llllll}67 & 50 & 50 & 17 & 83 & 100\end{array}$

All species in the library (177)

$\begin{array}{llllllllllllllllllllllllll}\text { a } & 68 & 64 & 70 & 74 & 70 & 67 & 83 & 87 & 73 & 72 & 80 & 80 & 75 & 72 & 88 & 90 & 73 & 69 & 72 & 72 & 69 & 68 & 84 & 88\end{array}$

$\begin{array}{llllllllllllllllllllllllllll}\text { b } & 1.56 & 1.58 & 1.64 & 1.73 & 1.65 & 1.65 & 1.92 & 1.96 & 1.7 & 1.7 & 1.73 & 1.82 & 1.75 & 1.78 & 2.01 & 2.05 & 1.58 & 1.57 & 1.64 & 1.72 & 1.67 & 1.63 & 1.94 & 2\end{array}$

Non-A. fumigatus species included in the library (92) a $\begin{array}{lllllllllllllllllllllllllllllllllll}53 & 50 & 57 & 61 & 56 & 51 & 71 & 77 & 55 & 55 & 70 & 70 & 59 & 57 & 78 & 83 & 60 & 54 & 59 & 58 & 53 & 51 & 71 & 78\end{array}$

$\begin{array}{llllllllllllllllllllllllll}\text { b } & 1.54 & 1.57 & 1.59 & 1.68 & 1.58 & 1.58 & 1.78 & 1.86 & 1.72 & 1.7 & 1.67 & 1.76 & 1.7 & 1.71 & 1.87 & 1.95 & 1.57 & 1.52 & 1.6 & 1.66 & 1.64 & 1.61 & 1.81 & 1.9\end{array}$

a: percentage of concordant identification, b: LS mean of the concordant identifications. 
Table 4 Modulation of the database performance for independent spots regarding the MSP creation parameters

\begin{tabular}{|c|c|c|c|c|c|c|c|c|}
\hline Libraries & $\begin{array}{l}\text { LS1 } \\
\text { mean }\end{array}$ & $\begin{array}{l}\mathrm{Nb} \text {. of } \\
\text { concordant } \\
\text { identifications }\end{array}$ & $\begin{array}{l}\text { LS1 mean of } \\
\text { concordant } \\
\text { identifications }\end{array}$ & $\begin{array}{l}\mathrm{Nb} \text {. of } \\
\text { non-concordant } \\
\text { identifications }\end{array}$ & $\begin{array}{l}\text { LS1 mean of } \\
\text { non -concordant } \\
\text { identifications }\end{array}$ & $\begin{array}{l}\text { Mean of the } \\
\text { difference between } \\
\text { LS1 and LS2 }\end{array}$ & $\begin{array}{l}\text { Max } \\
\text { frequency } \\
\text { parameter } \\
(\%)\end{array}$ & $\begin{array}{l}\mathrm{Nb} \text {. of } \\
\text { peaks } \\
\text { parameter }\end{array}$ \\
\hline B1 & 1.34 & 449 & 1.58 & 361 & 1.04 & 0.38 & 25 & 70 \\
\hline B1b & 1.34 & 449 & 1.58 & 361 & 1.04 & 0.38 & 25 & 100 \\
\hline B1C & 1.34 & 449 & 1.58 & 361 & 1.04 & 0.38 & 50 & 70 \\
\hline B1d & 1.36 & 473 & 1.60 & 337 & 1.03 & 0.40 & 50 & 100 \\
\hline B1e & 1.34 & 449 & 1.58 & 361 & 1.04 & 0.38 & 75 & 70 \\
\hline B1f & 1.32 & 445 & 1.56 & 365 & 1.03 & 0.36 & 75 & 100 \\
\hline B1g & 1.39 & 473 & 1.63 & 337 & 1.05 & 0.43 & 100 & 70 \\
\hline B1h & 1.39 & 473 & 1.63 & 337 & 1.05 & 0.43 & 100 & 100 \\
\hline B7 & 1.80 & 611 & 1.96 & 199 & 1.30 & 0.53 & 25 & 70 \\
\hline B7g & 1.80 & 595 & 1.96 & 215 & 1.35 & 0.50 & 100 & 70 \\
\hline
\end{tabular}

hyphae obtained in broth culture conditions [21-23]. Unfortunately, the more stringent the method, the less suited it is for high-throughput routine diagnoses. Furthermore, certain impediments are difficult to avoid in routine culture conditions, such as inter-technician variations, variation in protocol, and minor variations (temperature, humidity, or light), when aiming to standardize such protocols.

\section{Conclusion}

Overall, this study provides useful insight into architecture design of reference MS libraries utilized for the MALDI-TOF MS-based identification of filamentous fungi in routine clinical laboratories. Our results show that both incorporating an increased number of

Table 5 Multivariate analysis of the factors influencing the probability of the concordant identification of clinical isolates

\begin{tabular}{rlll}
\hline & OR & 95\% Confidence Interval & P \\
\hline Nb. raw spectra/RMS & & & \\
$\mathbf{4 0}$ vs. $\mathbf{1 0}$ & 1.1767 & 1.0503 to 1.3183 & 0.0050 \\
$\mathbf{4 0}$ vs. $\mathbf{2 0}$ & 1.2007 & 1.0705 to 1.3466 & 0.0018 \\
$\mathbf{2 0}$ vs. $\mathbf{1 0}$ & 0.9800 & 0.8933 to 1.0752 & 0.6698 \\
Nb. RMS/strain & & & \\
$\mathbf{4}$ vs. $\mathbf{1}$ & 1.3362 & 1.1929 to 1.4968 & $<10^{-4}$ \\
$\mathbf{4}$ vs. $\mathbf{2}$ & 1.1016 & 1.0122 to 1.1988 & 0.0250 \\
$\mathbf{2}$ vs. $\mathbf{1}$ & 1.2130 & 1.0950 to 1.3437 & 0.0002 \\
Nb. strains/species & & & \\
$\mathbf{3}$ vs. $\mathbf{1}$ & 1.2229 & 1.1173 to 1.3385 & $<10^{-4}$ \\
$\mathbf{3}$ vs. $\mathbf{2}$ & 1.0602 & 1.0095 to 1.1135 & 0.0193 \\
$\mathbf{2}$ vs. $\mathbf{1}$ & 1.1534 & 1.0683 to 1.2453 & 0.0003 \\
\hline
\end{tabular}

RMS: reference mass spectrum in the library constructed from several raw spectra. Nb.: number. subcultures from each strain and increasing the number of strains representing each species are key to improve the architecture of RMS libraries. These findings should be taken into account to construct a more effective library in clinical laboratories.

\section{Methods}

\section{Fungal strains}

The 90 reference filamentous fungus strains corresponding to 30 distinct species that were used to construct the eight distinct reference mass spectrum libraries are detailed in Table 6 . Of the 90 reference strains, 63 strains were graciously provided by the BCCM/IHEM (Belgian coordinated collection of microorganisms, Scientific Institute of Public Health, Mycology and Aerobiology Section, Brussels, Belgium), and 3 strains were provided by the Pasteur Institute (Paris, France). The remaining 24 strains were clinical isolates from the Marseille University Hospital mycology laboratory, which were accurately identified via DNA sequence analysis as described below. All strains used to construct the reference database are preserved in the BCCM/IHEM collection. The identification performance of each reference library was tested using 200 clinical isolates from the Marseille University Hospital mycology laboratory.

\section{Culture}

Each reference strain was subcultured on four Sabouraud Gentamicin Chloramphenicol agar plates (AES, France) at $30^{\circ} \mathrm{C}$. The strains used to construct the reference libraries and the isolates obtained from clinical samples were analyzed as soon as a fungal colony grew on the agar (usually after 48-72 hours). The clinical isolates were identified via morphological assessment, DNA sequencing, and MALDI-TOF MS as described below. 
Table 6 Details of the 90 reference strains corresponding to 30 filamentous fungi species of medical interest included in the reference libraries

\begin{tabular}{|c|c|c|c|}
\hline Species & Strain & Source & Included in library(ies) \\
\hline \multirow[t]{3}{*}{ Absidia corymbifera } & IHEM16288 & BCCM/IHEM & B0-B7 \\
\hline & IHEM14734 & BCCM/IHEM & B6-B7 \\
\hline & IHEM21658 & BCCM/IHEM & B7 \\
\hline \multirow[t]{3}{*}{ Acremonium strictum } & Pasteur 173 & Pasteur Institute & B7 \\
\hline & IHEM19179 & BCCM/IHEM & B0-B7 \\
\hline & IHEM 22371 & BCCM/IHEM & B6-B7 \\
\hline \multirow[t]{3}{*}{ Acrophialophora fusispora } & IHEM15939 & BCCM/IHEM & B0-B7 \\
\hline & IHEM19591 & BCCM/IHEM & B6-B7 \\
\hline & IHEM19730 & BCCM/IHEM & B7 \\
\hline \multirow[t]{3}{*}{ Alternaria alternata } & IHEM22669 & BCCM/IHEM & B6-B7 \\
\hline & IHEM21999 & BCCM/IHEM & B0-B7 \\
\hline & IHEM9788 & BCCM/IHEM & B7 \\
\hline \multirow[t]{3}{*}{ Aspergillus candidus } & IHEM15975 & BCCM/IHEM & B6-B7 \\
\hline & IHEM14607 & BCCM/IHEM & B7 \\
\hline & IHEM9678 & BCCM/IHEM & B0-B7 \\
\hline \multirow[t]{3}{*}{ Aspergillus flavus } & IHEM23376 & BCCM/IHEM & B6-B7 \\
\hline & IHEM14475 & BCCM/IHEM & B0-B7 \\
\hline & AFLA002 & clinical strain & B7 \\
\hline \multirow[t]{3}{*}{ Aspergillus fumigatus } & IHEM15161 & BCCM/IHEM & B0-B7 \\
\hline & IHEM19416 & BCCM/IHEM & B6-B7 \\
\hline & IHEM22145 & BCCM/IHEM & B7 \\
\hline \multirow[t]{3}{*}{ Aspergillus nidulans } & IHEM23179 & BCCM/IHEM & B0-B7 \\
\hline & IHEM23366 & BCCM/IHEM & B6-B7 \\
\hline & Pasteur 123 & Pasteur Institute & B7 \\
\hline \multirow[t]{3}{*}{ Aspergillus nigri section } & IHEM9673 & BCCM/IHEM & B0-B7 \\
\hline & IHEM5077 & BCCM/IHEM & B6-B7 \\
\hline & ANIG001 & clinical strain & B7 \\
\hline \multirow[t]{3}{*}{ Aspergillus terreus } & IHEM17777 & BCCM/IHEM & B0-B7 \\
\hline & IHEM18939 & BCCM/IHEM & B6-B7 \\
\hline & IHEM9995 & BCCM/IHEM & B7 \\
\hline \multirow[t]{3}{*}{ Beauveria bassiana } & IHEM6954 & BCCM/IHEM & B6-B7 \\
\hline & Pasteur 146 & Pasteur Institute & B7 \\
\hline & IHEM18747 & BCCM/IHEM & B0-B7 \\
\hline \multirow[t]{3}{*}{ Exophiala dermatitidis } & EXOP001 & clinical strain & B7 \\
\hline & IHEM23421 & BCCM/IHEM & B0-B7 \\
\hline & IHEM9780 & BCCM/IHEM & B6-B7 \\
\hline \multirow[t]{3}{*}{ Fusarium oxysporum } & IHEM18448 & BCCM/IHEM & B0-B7 \\
\hline & FUSA003 & clinical strain & B7 \\
\hline & IHEM20619 & BCCM/IHEM & B6-B7 \\
\hline \multirow[t]{3}{*}{ Fusarium solani } & IHEM22015 & BCCM/IHEM & B0-B7 \\
\hline & IHEM7504 & BCCM/IHEM & B6-B7 \\
\hline & 1000694 & clinical strain & B7 \\
\hline
\end{tabular}


Table 6 Details of the 90 reference strains corresponding to $\mathbf{3 0}$ filamentous fungi species of medical interest included in the reference libraries (Continued)

\begin{tabular}{|c|c|c|c|}
\hline \multirow[t]{3}{*}{ Fusarium verticillioides } & IHEM20180 & BCCM/IHEM & B6-B7 \\
\hline & IHEM22962 & BCCM/IHEM & B7 \\
\hline & IHEM18495 & BCCM/IHEM & BO-B7 \\
\hline \multirow[t]{3}{*}{ Microsporum audouinii } & 013 & clinical strain & BO-B7 \\
\hline & 027 & clinical strain & B6-B7 \\
\hline & 169 & clinical strain & B7 \\
\hline \multirow[t]{3}{*}{ Microsporum canis } & $05-05-00921$ & clinical strain & BO-B7 \\
\hline & 249 & clinical strain & B6-B7 \\
\hline & 1100085 & clinical strain & B7 \\
\hline \multirow[t]{3}{*}{ Microsporum gypseum } & 05-05-00961 & clinical strain & BO-B7 \\
\hline & $\mathrm{MO}$ & clinical strain & B6-B7 \\
\hline & 93629 & clinical strain & B7 \\
\hline \multirow[t]{3}{*}{ Paecilomyces variotii } & IHEM17703 & BCCM/IHEM & B6-B7 \\
\hline & IHEM3285 & BCCM/IHEM & B7 \\
\hline & IHEM16627 & BCCM/IHEM & BO-B7 \\
\hline \multirow[t]{3}{*}{ Penicillium aurantiogriseum } & IHEM18723 & BCCM/IHEM & BO-B7 \\
\hline & IHEM20176 & BCCM/IHEM & B6-B7 \\
\hline & IHEM20357 & BCCM/IHEM & B7 \\
\hline \multirow[t]{3}{*}{ Penicillium chrysogenum } & IHEM17894 & BCCM/IHEM & BO-B7 \\
\hline & IHEM22667 & BCCM/IHEM & B6-B7 \\
\hline & IHEM20859 & BCCM/IHEM & B7 \\
\hline \multirow[t]{3}{*}{ Rhizomucor pusillus } & IHEM21236 & BCCM/IHEM & BO-B7 \\
\hline & IHEM18686 & BCCM/IHEM & B6-B7 \\
\hline & IHEM16462 & BCCM/IHEM & B7 \\
\hline \multirow[t]{3}{*}{ Rhizopus oryzae } & IHEM13186 & BCCM/IHEM & BO-B7 \\
\hline & IHEM21660 & BCCM/IHEM & B7 \\
\hline & IHEM16287 & BCCM/IHEM & B6-B7 \\
\hline \multirow[t]{3}{*}{ Scedosporium apiospermum } & IHEM3817 & BCCM/IHEM & B7 \\
\hline & IHEM6908 & BCCM/IHEM & B6-B7 \\
\hline & IHEM14632 & BCCM/IHEM & B0-B7 \\
\hline \multirow[t]{3}{*}{ Scedosporium prolificans } & IHEM5739 & BCCM/IHEM & BO- \\
\hline & IHEM18755 & BCCM/IHEM & B6 \\
\hline & IHEM22339 & BCCM/IHEM & B7 \\
\hline \multirow[t]{3}{*}{ Scopulariopsis brevicaulis } & IHEM15574 & BCCM/IHEM & B0-B7 \\
\hline & IHEM1690 & BCCM/IHEM & B6 \\
\hline & IHEM22982 & BCCM/IHEM & B7 \\
\hline \multirow[t]{3}{*}{ Trichophyton mentagrophytes } & 1036279 & clinical strain & BO-B7 \\
\hline & 1039316 & clinical strain & \\
\hline & $5-97-00858$ & clinical strain & B7 \\
\hline \multirow[t]{3}{*}{ Trichophyton rubrum } & $60 D$ & clinical strain & BO- \\
\hline & 184 & clinical strain & B6-B7 \\
\hline & 235 & clinical strain & B7 \\
\hline
\end{tabular}


Table 6 Details of the 90 reference strains corresponding to $\mathbf{3 0}$ filamentous fungi species of medical interest included in the reference libraries (Continued)

\begin{tabular}{|c|c|c|c|}
\hline \multirow[t]{3}{*}{ Trichophyton roseum } & IHEM1535 & BCCM/IHEM & BO-B7 \\
\hline & 7941 & clinical strain & B6-B7 \\
\hline & IHEM2478 & BCCM/IHEM & B7 \\
\hline \multirow[t]{3}{*}{ Trichophyton soudanense } & 243 & clinical strain & BO-B7 \\
\hline & 095 & clinical strain & B6-B7 \\
\hline & 1100082 & clinical strain & B7 \\
\hline
\end{tabular}

BCCM/IHEM: Scientific Institute of Public Health, Mycology \& Aerobiology Section, Brussels, Belgium; the characteristics of the mass spectra libraries B0 to B7 are described in Table 7.

\section{Clinical isolate identification}

All 200 clinical isolates were identified in parallel by two trained mycologists following the identification keys of the Atlas of Clinical Fungi [24]. If the morphological identification was impossible or conflicted with the MALDI-TOF MS-based identification results, the isolate was further analyzed using DNA sequencing. DNA sequence-based identification was performed by analyzing the ITS 2 (primer ITS3: GCA TCG ATG AAG AAC GCA GC and primer ITS4c: TCC TCC GCT TAT TGA TAT GC) and D1-D2 (primer D1: AAC TTA AGC ATA TCA ATA AGC GGA GGA and primer D2: GGT CCG TGT TTC AAG ACG G) variable regions of the $28 \mathrm{~S}$ unit of the rRNA gene as described by de Hoog et al. [24]. DNA extraction was performed using a QIAmp DNA kit (QIAGEN, Courtaboeuf, France). The reaction mixture was subjected to 35 cycles of $30 \mathrm{~s}$ denaturation at $94^{\circ} \mathrm{C}, 30 \mathrm{~s}$ primer annealing at $53^{\circ} \mathrm{C}$, and $1 \mathrm{~min}$ primer extension at $72^{\circ} \mathrm{C}$ for the ITS 2 region and 40 cycles of $20 \mathrm{~s}$ denaturation at $94^{\circ} \mathrm{C}, 30 \mathrm{~s}$ primer annealing at $58^{\circ} \mathrm{C}$, and $1 \mathrm{~min}$ primer extension at $72^{\circ} \mathrm{C}$ for the D1-D2 region. The sequencing reactions were performed using the same primers used for amplification. In both cases, the sequencing mixture was subjected to 25 cycles of 10 s denaturation at $96^{\circ} \mathrm{C}, 5 \mathrm{~s}$ primer annealing at $50^{\circ} \mathrm{C}$, and 4 min primer extension at $60^{\circ} \mathrm{C}$. Purification of the sequences was performed using BigDye ${ }^{\circledR}$ XTerminator ${ }^{\mathrm{mat}}$ (Applied Biosystems, Inc., Courtaboeuf, France), and the different reactions were processed using a 3130 Genetic Analyzer (Applied Biosystems, Inc., Courtaboeuf, France). The resulting sequences were then compared using the Medical Fungi pairwise sequence alignment tool (http://www.cbs.knaw.nl/Medical/BioloMICSSequen ces.aspx). Identification was validated when the sequence was at least 300 nucleotides long and the similarity percentage was over $98 \%$.

\section{Raw mass spectra acquisition}

The colonies were gently scraped with sterile plastic pliers to obtain an aliquot (approximately 3-4 $\mathrm{mm}$ in diameter) of fungal spores and hyphae. This sample was first suspended in $75 \%$ ethanol HPLC. Next, the hydroalcoholic solution was removed via $10 \mathrm{~min}$ centrifugation at 13,000 g, and the pellet was suspended in $10 \mu \mathrm{L}$ of $70 \%$ formic acid (Sigma-Aldrich, France) by vigorously pipetting the sample up and down. After a 5-min incubation, $10 \mu \mathrm{L}$ of acetonitrile HPLC (VWR International S.A.S., Fontenay-sous-Bois, France) was added, and the mixture was incubated at room temperature for $5 \mathrm{~min}$. Finally, the sample was centrifuged for $2 \mathrm{~min}$ at $13,000 \mathrm{~g}$. One microliter of the supernatant (consisting of a mixture of fungal proteins) was deposited for each reference strain subculture in 10 replicates on a polished steel target (MTP384, Bruker Daltonics GmbH, Bremen, Germany) and air-dried. Each deposit was then covered with $1 \mu \mathrm{L}$ of a freshly prepared solution of $\alpha$-cyano-4-

Table 7 Architectural characteristics of the reference libraries

\begin{tabular}{|c|c|c|c|c|}
\hline Library number & $\begin{array}{l}\text { Number of raw spectra } \\
\text { per RMS }\end{array}$ & $\begin{array}{l}\text { Number of RMS } \\
\text { per strain }\end{array}$ & $\begin{array}{l}\text { Number of strains } \\
\text { per species }\end{array}$ & Library characteristics \\
\hline BO & 4 & 1 & 1 & 1 RMS4 $\times 30$ strains \\
\hline B1 & 10 & 1 & 1 & 1 RMS10 $\times 30$ strains \\
\hline B2 & 10 & 2 & 1 & 2 RMS10 $\times 30$ strains \\
\hline B3 & 10 & 4 & 1 & 4 RMS10 $\times 30$ strains \\
\hline B4 & 20 & 2 & 1 & 2 RMS20 $\times 30$ strains \\
\hline B5 & 40 & 1 & 1 & 1 RMS40 $\times 30$ strains \\
\hline B6 & 10 & 4 & 2 & 4 RMS10 $\times 60$ strains \\
\hline B7 & 10 & 4 & 3 & 4 RMS10 $\times 90$ strains \\
\hline
\end{tabular}


hydroxycinnamic acid (HCCA) in 50\% acetonitrile HPLC (VWR International S.A.S., Fontenay-sous-Bois, France) and $2.5 \%$ trifluoroacetic acid HPLC (TFA) matrix (Applied Biosystems ${ }^{\circledR}$, Villebon sur yvette, France) [21]. The spectra were acquired after 650 shots in linear mode using an UltrafleXtreme ${ }^{\mathrm{TM}}$ instrument (Bruker Daltonics, Germany) in the ion-positive mode with a 337-nm nitrogen laser. The following adjustments were used: delay, $170 \mathrm{~ns}$; ion source 1 voltage, $20 \mathrm{kV}$; ion source 2 voltage, $18.5 \mathrm{kV}$; mass range, 3-20 kDa; and measuring raster: spiral_small. An E. coli calibration was performed before every experiment using a Bruker Bacterial Test Standard (Bruker Daltonics GmbH, Bremen, Germany). The data were automatically acquired using the AutoXecute function of the FlexControl v2.4 software and then exported into MALDI Biotyper v2.1 (Bruker Daltonics) software. Only the peaks with a signal/noise ratio $\geq 10$ were considered.

\section{Constructing the reference mass spectra (RMS)}

The RMS were established by combining i) 4 raw spectra obtained from one subculture (RMS4); ii) 10 raw spectra obtained from one subculture (RMS10); iii) 20 raw spectra, 10 from two subcultures each (RMS20); or iv) 40 raw spectra, 10 from four subcultures each (RMS40) of a given reference strain using the "MSP creation" function of the MALDI Biotyper v2.1 software (Table 7). The following settings were applied (Bruker's default parameters): Max. Mass Error of each single spectrum: 2000; Desired Mass Error of the MSP: 200; Desired Peak Frequency Minimum: 25\%; and Max. Desired Peak Number of the MSP: 70. The modulation of the number of peaks and desired peak frequency minimum of the MSP creation parameters has been tested regarding the $\mathrm{B} 1$ library, and the modified parameters were tested on the B7 database (Table 4).

\section{Architecture of the eight mass spectral libraries}

The same fungal species were included in the eight libraries that differed in number of raw spectra used to build the RMS (described above), RMS included for each reference strain, and strains included. The characteristics of the various libraries are detailed in Table 2 .

\section{MALDI-TOF MS-based identification of clinical isolates}

Raw mass spectra were obtained from clinical isolates using the same procedure as for the reference strains with the exception that the supernatant were deposited in quadruplicate. The deposits, referred to as spots 1,2 , 3 , and 4, correspond to the first, second, third, and fourth extraction supernatant deposit of each sample, respectively. The raw MS data for each spot was successively matched to the eight reference libraries, and the resulting "best match" LS values were calculated using MALDI Biotyper software. An alternate identification process was assessed by constructing an MSP with the four spots corresponding to each of the clinical isolates and comparing isolate MSP with each of the RMS in the libraries.

The interpretation of the results was initially performed independently of the LS value. If the MS identification was identical to the microscopic identification or the sequencing analysis results, the identification was considered concordant, regardless of the LS value; otherwise, it was considered a non-concordant identification. Next, the LS value was considered to be applicable in comparing the performance of the various libraries. As approximately half of the clinical isolates corresponded to the Aspergillus fumigatus species, a comparison was also performed between the libraries when either considering or disregarding this dominant species.

Library performance was also compared regarding the method by which the clinical quadruplicates were considered as follows: i) each spectrum was treated independently, ii) only the spectrum with the highest LS was taken into account, regardless of whether it was concordant, and iii) an MSP of the four spectra was constructed, and the clinical MSP was compared to each library.

\section{Ambiguous MS identifications}

Some of the species included in this study are known to be difficult to distinguish, even via ITS sequencing. Reference spectra were included in the libraries, but concordance could neither be confirmed nor contradicted. The species included were Penicillium aurantiogriseum and Penicillium chrysogenum. Both MS identifications were then considered concordant with the other identification methods.

\section{Reference mass spectra library architecture assessment}

Analyzing 200 clinical isolates, we tested the influence of the number of the following parameters on identification effectiveness: i) raw spectra used to build a reference MS, ii) reference $\mathrm{MS}$ included per strain, and iii) strains per species included in the library. The various reference spectrum architectures were compared with respect to the number of correct and false identifications as well as the mean LS values of both correct and false identifications.

\section{Statistical analysis}

The concordant and non-concordant identification results were compared two by two using the paired and non-parametric McNemar's test. The results of the quantitative variable LS analysis were compared using the non-parametric rank sum test of the Kruskall-Wallis test. When the results of the Kruskall-Wallis test indicated a statistical difference between the LS values 
derived from the different mass spectral libraries, a post hoc statistical analysis was performed, which involved a pairwise comparison of the LS values obtained from each library using the Wilcoxon signed-rank test with Bonferroni adjustment. These analyses were performed using R software (http://www.r-project.org/) with the MASS and ROCR packages. To further examine the influence of library architecture on the probability of obtaining a correct identification, a multivariate analysis was conducted with the Genmod procedure of the SAS 9.2 (Cary, NC, USA) statistical software using the generalized estimating equations option to account for the non-independence of identification results obtained from the same isolate tested against distinct libraries. These analyses were performed to identify the optimal reference library architecture; therefore, the results obtained with isolates for which the species was not included in the library were excluded from this multivariate analysis. All statistical tests were two-sided with a $\mathrm{p} \leq 0.05$ significance level.

\section{Availability of supporting data}

These data are included in Table 6 entitled "Details of the 90 reference strains included in the reference libraries".

\begin{abstract}
Abbreviations
MALDI-TOF: Matrix-assisted laser desorption/ionization time-of-flight; MS: Mass Spectrometry; RMS: Reference Meta Spectrum; MSP: Meta spectrum; LS: Log Score; PCR: Polymerase Chain Reaction; DNA: Deoxyribonucleic acid; rRNA: Ribosomal ribonucleic acid; s: Second; ${ }^{\circ} \mathrm{C}$ : Temperature in Celsius; min: Minute; $\mu \mathrm{L}$ : Microliter; $\mathrm{nm}$ : Nanometer; ns: Nanosecond; kV: Kilovolt; kDa: Kilodalton; MSL: Mass spectra library; Nb.: Number.
\end{abstract}

\section{Competing interests}

The authors declare that they have no competing interests.

\section{Authors' contributions}

$A C N, C C, C L, P F, S R$, and $M H$ performed the experiments. ACN, CC, SR, and RP conceived the study, analyzed the data, and wrote the manuscript. CC and SR carried out the statistical analyses. ACN and CC prepared the figures and tables. All authors read and approved the final manuscript.

\section{Acknowledgements}

We thank the Pasteur Institute of Paris, France and the BCCM/IHEM public collection of Brussels, Belgium for kindly providing the reference strains. We also thank Sandra Moore for correcting the manuscript.

\section{Author details \\ ${ }^{1}$ AP-HM, Parasitologie-Mycologie, CHU Timone, 13005, Marseille, France. ${ }^{2}$ Aix-Marseille Université, UMR MD3, 13005, Marseille, France. ${ }^{3}$ Service Proteomique, Centre d'Immunologie de Marseille Luminy, 13009, Marseille, France. ${ }^{4}$ BCCM/IHEM: Scientific Institute of Public Health, Mycology and Aerobiology Section, Brussels, Belgium.}

Received: 24 December 2012 Accepted: 25 March 2013 Published: 8 April 2013

\section{References}

1. Balajee SA, Nickle D, Varga J, Marr KA: Molecular studies reveal frequent misidentification of Aspergillus fumigatus by morphotyping. Eukaryotic Cell 2006, 5:1705-1712.
2. Samson RA, Hong S, Peterson SW, Frisvad JC, Varga J: Polyphasic taxonomy of Aspergillus section Fumigati and its teleomorph Neosartorya. Stud. Mycol. 2007, 59:147-203.

3. Baker SE: Aspergillus niger genomics: past, present and into the future. Med. Mycol 2006, 44(1):17-21.

4. Bennett JW, In Aspergillus: Molecular Biology and Genomics: An Overview of the Genus Aspergillus. Caister Academic Press: edited by Machida M, Gomi K; 2010:1-17.

5. Alexander BD: Diagnosis of fungal infection: new technologies for the mycology laboratory. Transpl Infect Dis 2002, 4(Suppl 3):32-37.

6. Lau A, Chen S, Sleiman S, Sorrell T: Current status and future perspectives on molecular and serological methods in diagnostic mycology. Future Microbiology 2009, 4:1185-1222.

7. Croxatto A, Prod'hom G, Greub G: Applications of MALDI-TOF mass spectrometry in clinical diagnostic microbiology. FEMS Microbiol. Rev. 2012, 36:380-407.

8. Marvin LF, Roberts MA, Fay LB: Matrix-assisted laser desorption/ionization time-of-flight mass spectrometry in clinical chemistry. Clin. Chim. Acta 2003, 337:11-21.

9. Seyfarth F, Ziemer M, Sayer HG, Burmester A, Erhard M, Welker M, Schliemann S, Straube E, Hipler U-C: The use of ITS DNA sequence analysis and MALDI-TOF mass spectrometry in diagnosing an infection with Fusarium proliferatum. Exp. Dermatol. 2008, 17:965-971.

10. Kemptner J, Marchetti-Deschmann M, Mach R, Druzhinina IS, Kubicek CP, Allmaier G: Evaluation of matrix-assisted laser desorption/ionization (MALDI) preparation techniques for surface characterization of intact Fusarium spores by MALDI linear time-of-flight mass spectrometry. Rapid Commun. Mass Spectrom. 2009, 23:877-884.

11. Marinach-Patrice C, Lethuillier A, Marly A, Brossas J-Y, Gené J, Symoens F, Datry A, Guarro J, Mazier D, Hennequin C: Use of mass spectrometry to identify clinical Fusarium isolates. Clin. Microbiol. Infect. 2009, 15:634-642.

12. Erhard M, Hipler U-C, Burmester A, Brakhage AA, Wöstemeyer J: Identification of dermatophyte species causing onychomycosis and tinea pedis by MALDI-TOF mass spectrometry. Exp. Dermatol. 2008, 17:356-361.

13. L'Ollivier C, Cassagne C, Normand A-C, Bouchara J-P, Contet-Audonneau M, Hendricks M, Fourquet P, Coulibaly O, Piarroux R, Ranque S: A MALDI-TOF MS procedure for clinical dermatophyte species identification in the routine laboratory. Medical Mycology 2013. doi:10.3109/ 13693786.2013.781691. ID: 781691.

14. Li TY, Liu BH, Chen YC: Characterization of Aspergillus spores by matrixassisted laser desorption/ionization time-of-flight mass spectrometry. Rapid Commun. Mass Spectrom. 2000, 14:2393-2400.

15. Alanio A, Beretti J-L, Dauphin B, Mellado E, Quesne G, Lacroix C, Amara A, Berche $P$, Nassif $X$, Bougnoux M-E: Matrix-assisted laser desorption ionization time-of-flight mass spectrometry for fast and accurate identification of clinically relevant Aspergillus species. Clin. Microbiol. Infect. 2011, 17:750-755.

16. Coulibaly O, Marinach-Patrice C, Cassagne C, Piarroux R, Mazier D, Ranque S: Pseudallescheria/Scedosporium complex species identification by Matrix-Assisted Laser Desorption Ionization Time-Of-Flight Mass Spectrometry. Med. Mycol. 2011, 49:621-626.

17. Chen H-Y, Chen Y-C: Characterization of intact Penicillium spores by matrix-assisted laser desorption/ionization mass spectrometry. Rapid Commun. Mass Spectrom. 2005, 19:3564-3568.

18. Hettick JM, Green BJ, Buskirk AD, Kashon ML, Slaven JE, Janotka E, Blachere FM, Schmechel D, Beezhold DH: Discrimination of Penicillium isolates by matrix-assisted laser desorption/ionization time-of-flight mass spectrometry fingerprinting. Rapid Commun. Mass Spectrom. 2008, 22:2555-2560.

19. Tao J, Zhang G, Jiang Z, Cheng Y: Feng J. Chen Z: Detection of pathogenic Verticillium spp. using matrix-assisted laser desorption/ionization time-of-flight mass spectrometry. Rapid Commun. Mass Spectrom. 2009, 23:3647-3654.

20. DE Respinis S, Vogel G, Benagli C, Tonolla M, Petrini O, Samuels G: MALDITOF MS of Trichoderma: a model system for the identification of microfungi. Mycological Progress 2010, 9:79-100.

21. Cassagne $C$, Ranque $S$, Normand A-C, Fourquet P, Thiebault S, Planard C, Hendrickx M, Piarroux R: Mould routine identification in the clinical laboratory by matrix-assisted laser desorption ionization time-of-flight mass spectrometry. PLOS ONE 2011, 6:e28425.

22. De Carolis E, Posteraro B, Lass-Flörl C, Vella A, Florio AR, Torelli R, Girmenia C, Colozza C, Tortorano AM, Sanguinetti M, Fadda G: Species identification 
of Aspergillus, Fusarium and Mucorales with direct surface analysis by matrix-assisted laser desorption ionization time-of-flight mass spectrometry. Clin. Microbiol. Infect. 2012, 18:475-484.

23. Oda K, Kakizono D, Yamada O, lefuji H, Akita O, Iwashita K: Proteomic analysis of extracellular proteins from Aspergillus oryzae grown under submerged and solid-state culture conditions. Appl. Environ. Microbiol. 2006, 72:3448-3457.

24. Atlas of Clinical Fungi: Atlas of Clinical Fungi. 2nd edition. ASM Press; 2001.

\section{doi:10.1186/1471-2180-13-76}

Cite this article as: Normand et al:: Assessment of various parameters to improve MALDI-TOF MS reference spectra libraries constructed for the routine identification of filamentous fungi. BMC Microbiology 2013 13:76

\section{Submit your next manuscript to BioMed Central and take full advantage of:}

- Convenient online submission

- Thorough peer review

- No space constraints or color figure charges

- Immediate publication on acceptance

- Inclusion in PubMed, CAS, Scopus and Google Scholar

- Research which is freely available for redistribution 